\title{
THE FULLY AUTOMATED MELTSHOP: BETTER SAFETY, CONTROL AND SAVINGS*
}

John Anthony Vasquez ${ }^{1}$

Francesco Memoli

\begin{abstract}
During the last years Riva Acciaio Verona has taken the decision to invest in the route of a fully automated meltshop. The aim is to reduce at the minimum the operator's intervention from the bucket preparation till the ladle transfer to the continuous casting bay. The foundation for achieving the goal is the introduction of innovative technological solutions (fully automated cranes for bucket charge in the EAF, robots for sampling, etc. The paper underlines how the different solutions are based on the best practices to improve operator's safety and describes the different steps of the project, the different solutions adopted and the results in terms of production, energy savings, cost reduction and improved safety. Details are presented for: scrap yard management and bucket preparation, EAFs centralized controls and related robots for auxiliary operations (sampling, EBT cleaning, scraper for slag door automation, complete mass \& energy balance based on process control), LFs centralized controls and process integration.

Keywords: Fully automated melt shop; Operator safety; Process optimization; Economic benefits.
\end{abstract}

1 MSc Engineering Materials, BEng Metallurgy, Technical Service Manager/ Steelmaking, Tenova Core, Pittsburgh, USA.

2 MS Engineering, BEng Mechanical, Executive VP of the Metals Commercial Network, Tenova Core, Pittsburgh, USA. 


\section{INTRODUCTION}

During the last few years, some steel plants, among which Riva in Verona, Italy, have taken the decision to invest in the route of a fully automated Meltshop. The aim is to reduce at the minimum the operator's intervention from the bucket preparation till the ladle transfer to the continuous casting bay. The foundation for achieving the goal is the introduction of innovative technological solutions: fully automated cranes for bucket charge in the EAF, robots for sampling, large use of CCTV in the Meltshop, automatic tapping devices, automatic sanding devices for EBT, etc.

This paper underlines how the different solutions are based on the best practices to improve operator's safety and describes the different steps of the project, the different solutions adopted and the results in terms of safety enhancement, production increase, energy savings and, more in general, cost reduction.

Details are presented for: scrap yard management and bucket preparation, EAFs centralized controls and related robots for auxiliary operations (sampling, EBT cleaning, scraper for slag door automation, complete mass \& energy balance based on process control), centralized controls and process integration.

\subsection{EAF Control Room with no Windows}

Commonly control rooms and pulpits have large glass windows to allow visibility over certain EAF operations that require a clear field of view between the operator controls and the EAF, such as: monitoring the foaming slag through the slag door, charging the bucket, slipping the electrodes, tapping the EAF, etc.

These windows, even when made out of shock resistant materials or with protective steel bars, tend to crack or break - generating a safety hazard - due to the nature of the operations and tough environment. The current state of technology in remote sensing, monitoring, cameras and automation allows the following:

- Full plant sights remote high resolution cameras and monitors

- Huge panels of operators guidance

- Desk shaped pulpits with only electrodes command joysticks and emergency push buttons

- Touch screen monitors and operator panels ergonomically located.

Therefore, the control rooms can be fully enclosed, with no windows exposed to the EAF's harsh environment that provide the following benefits:

- Location of the pulpit far from the furnace and possibly outside the EAF building

- Blast effects free

- Noise free

- Dirt and dust free

- Reduce number of operators with remote tapping from the main control pulpit. 

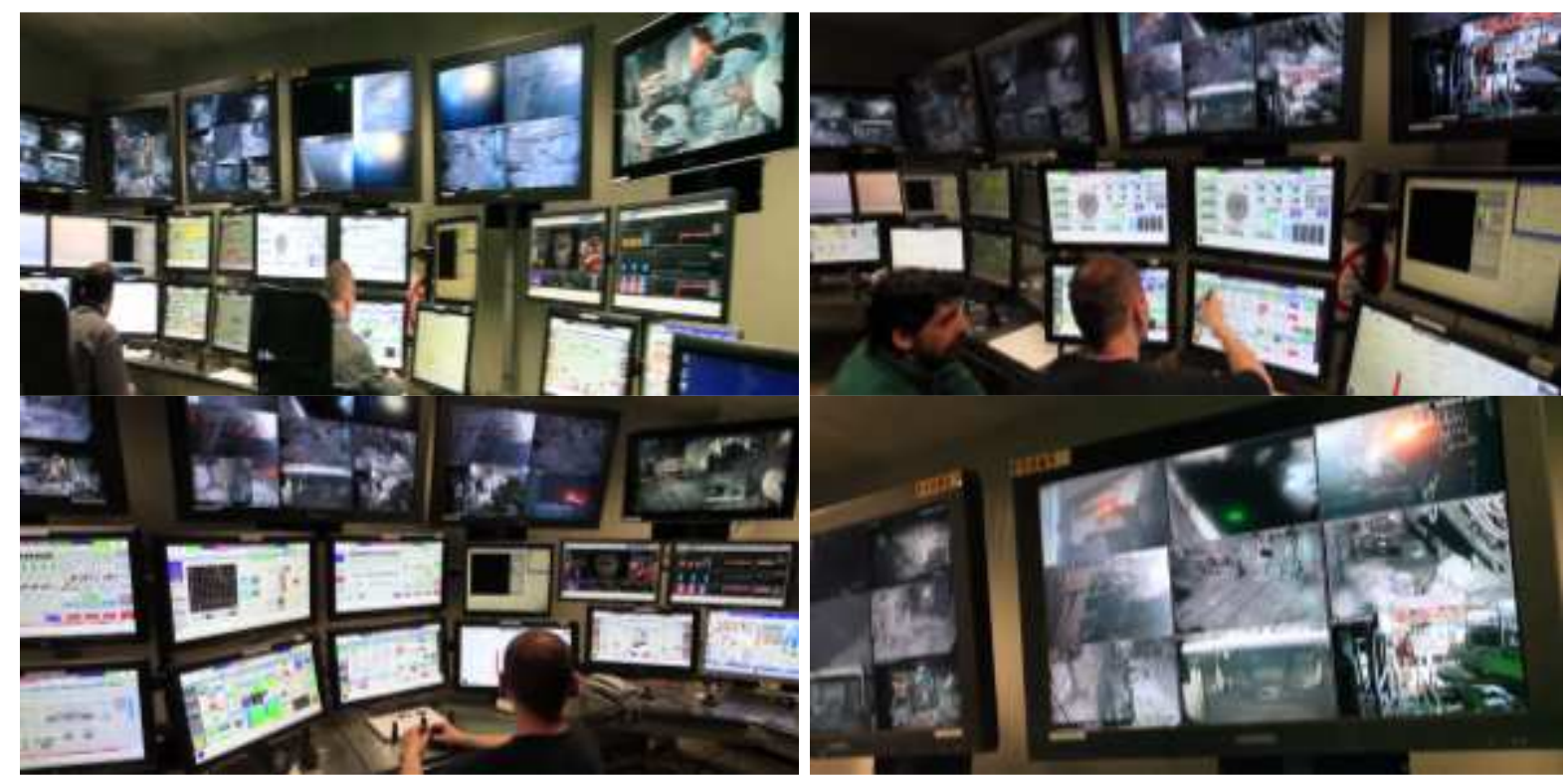

Figure 1 - The Modern EAF control room with no exposed windows to the EAF or operating floor

As a matter of fact, these type of control rooms can have more or less CCTV cameras or HMI screens depending on the number of equipment that the operator have to supervise.

In some cases these rooms are located just outside the building, but still close to the area where the equipment is located, so that in case of need the operator has easy access to the equipment, but during normal operation he is totally protected.

\subsection{Robotized Temperature and Sampling}

Temperature and sampling devices have existed for many years, however the introduction of a programmable and customizable devices that allows for many degrees of free-movement results in the automation of the 2 activities that previous systems could not, automatically load the thermocouple or chemical sampling cartridges and discharge them once used.

This literally means that no operator needs to stand next to the "old" sampling lance to perform the operation that is now completely controllable from an enclosed pulpit, enhancing safety. The many degrees of free movement on these devices/robots also ensure full penetration into the furnace, regardless of how small the slag door is.

Also repetitiveness and accuracy of the readings is achieved, as it can control its immersion depending on the angle of the furnace and bath level. 

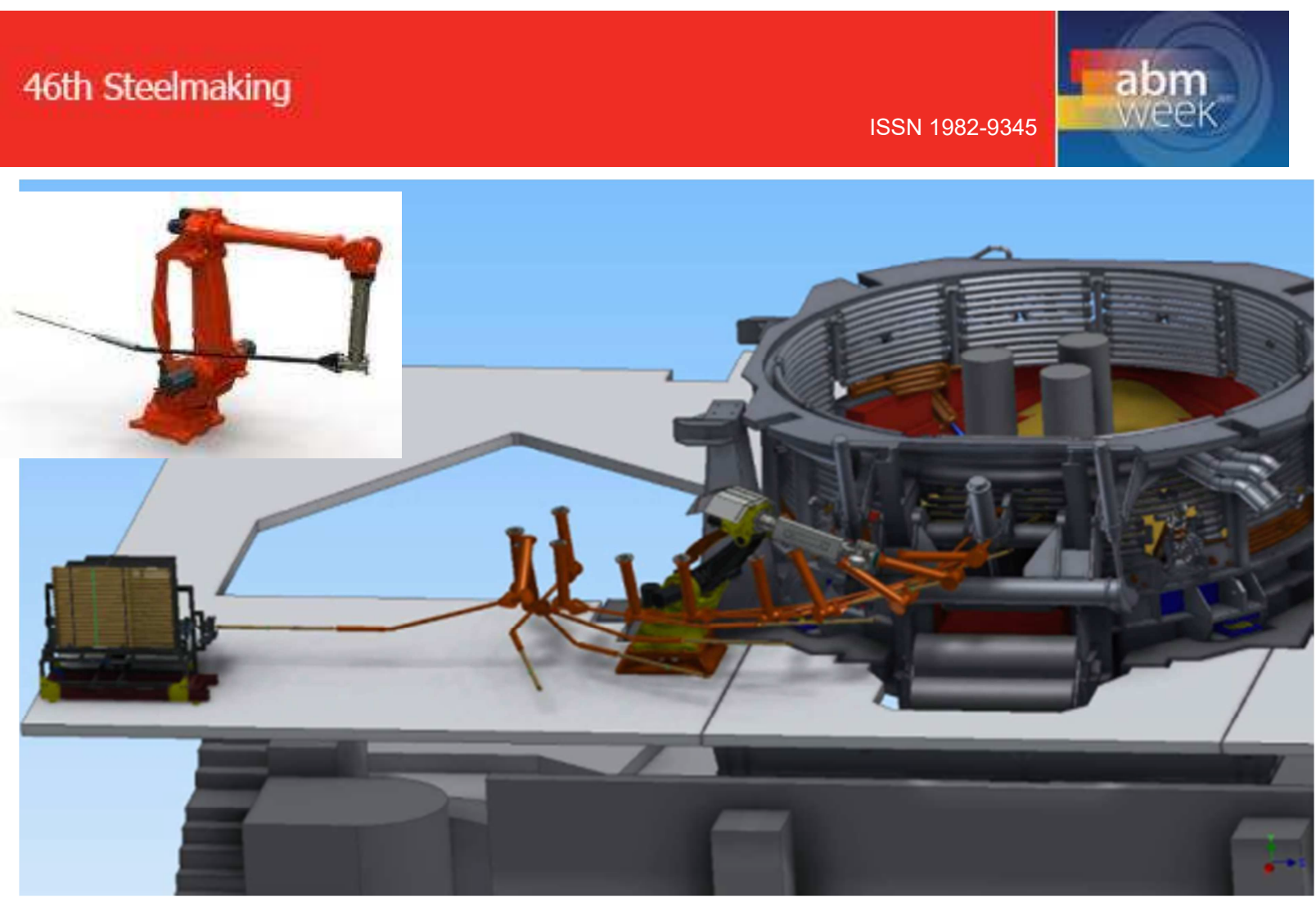

Figure 2 - Robotic sampling device can load and discharge cartridges automatically

\subsection{TAT Automatic Tapping}

The TAT system is comprised of several individual and customizable sub-systems that completely automate the different operations required to tap the furnace at the end of the melting phase.

This involves: tilting the furnace during tapping operation, opening the EBT flap, controlling the angle of the EAF throughout the tapping of the liquid steel, fast backtilting, remote inspection the EBT after tapping through CCTV system and remote sanding of the EBT prior to the next heat.

Automated EBT free opener: In the event of a non-free opening, an oxygen pipe is available to lance the EBT from the opening on the sump-panel. Usually this operation involved in the past a man who had to get access to the bottom of the EBT with the flap open, lancing oxygen from the bottom using a bent pipe and trying to free up the sand to let the liquid steel flow out of the EBT hole. This was and in general still is one of the most dangerous operations in the EAF due to the short distance between the man and the liquid steel, which is eventually flowing out of the EAF sometimes in an uncontrolled way. Thanks to the new automated device, the man is removed from this operation. 


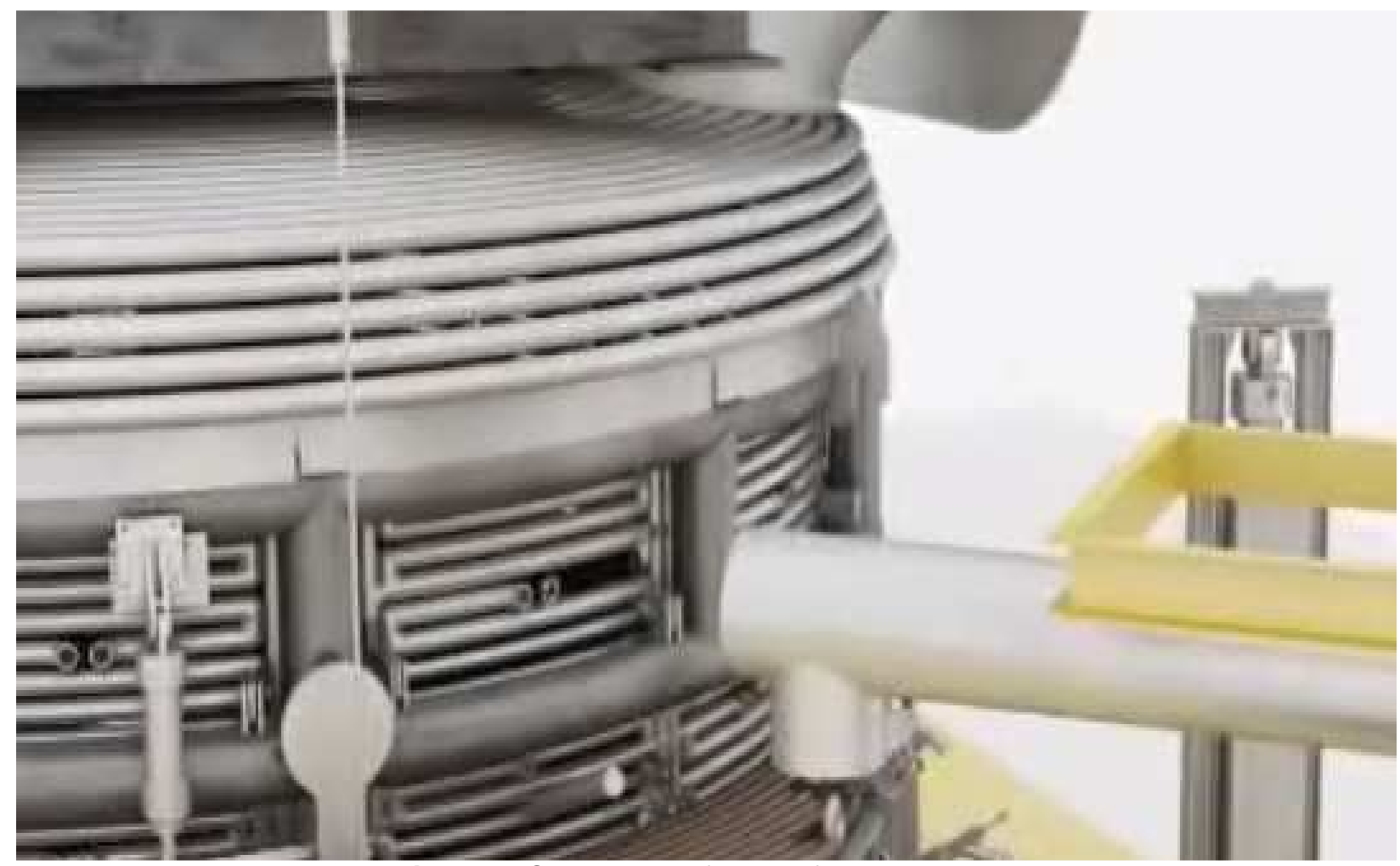

Figure 3. Oxygen Pipe for EBT free opening

EBT Cleaner: After tapping, in case an obstruction of the EBT is detected thanks to the CCTV inspection camera, another device - EBT cleaner - can be deployed to clean the obstruction from the bottom of the EAF, always in remote mode.
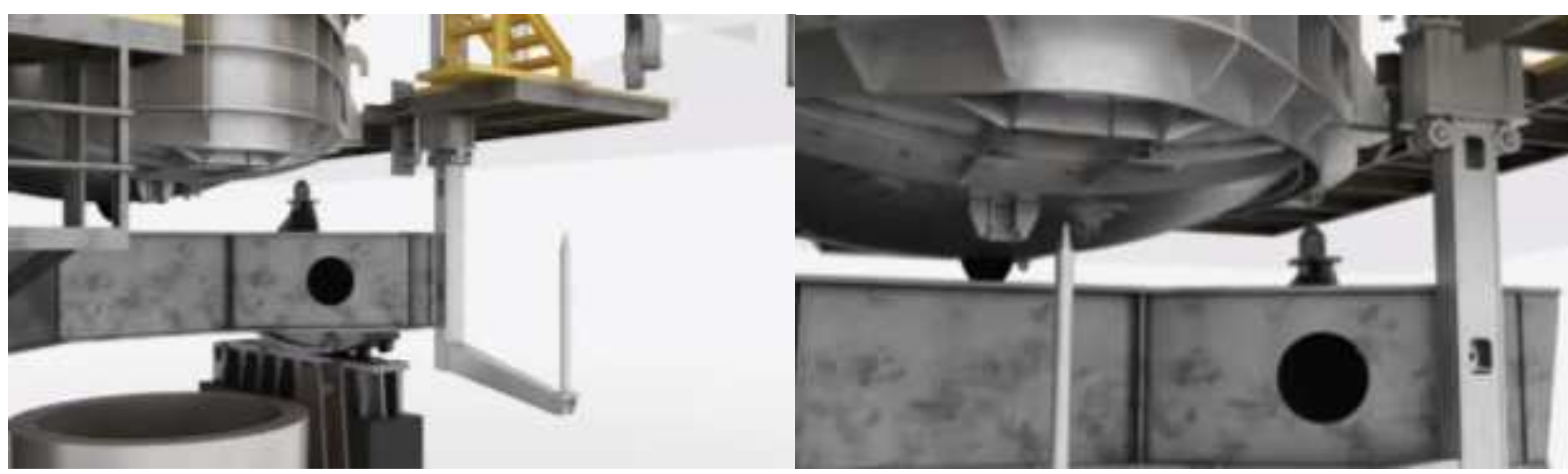

Figure 4. EBT Cleaner

Remote tapping to minimize slag pouring (Tenova TAT): A combination of images from infrared and standard video cameras located on strategic locations near the EBT, water cooled and shielded to protect them against heat and slag splashes, together with inputs such as steel weight in the ladle, steel weight in the furnace and proprietary software, are combined to automatically set the tilting angle of the furnace. 

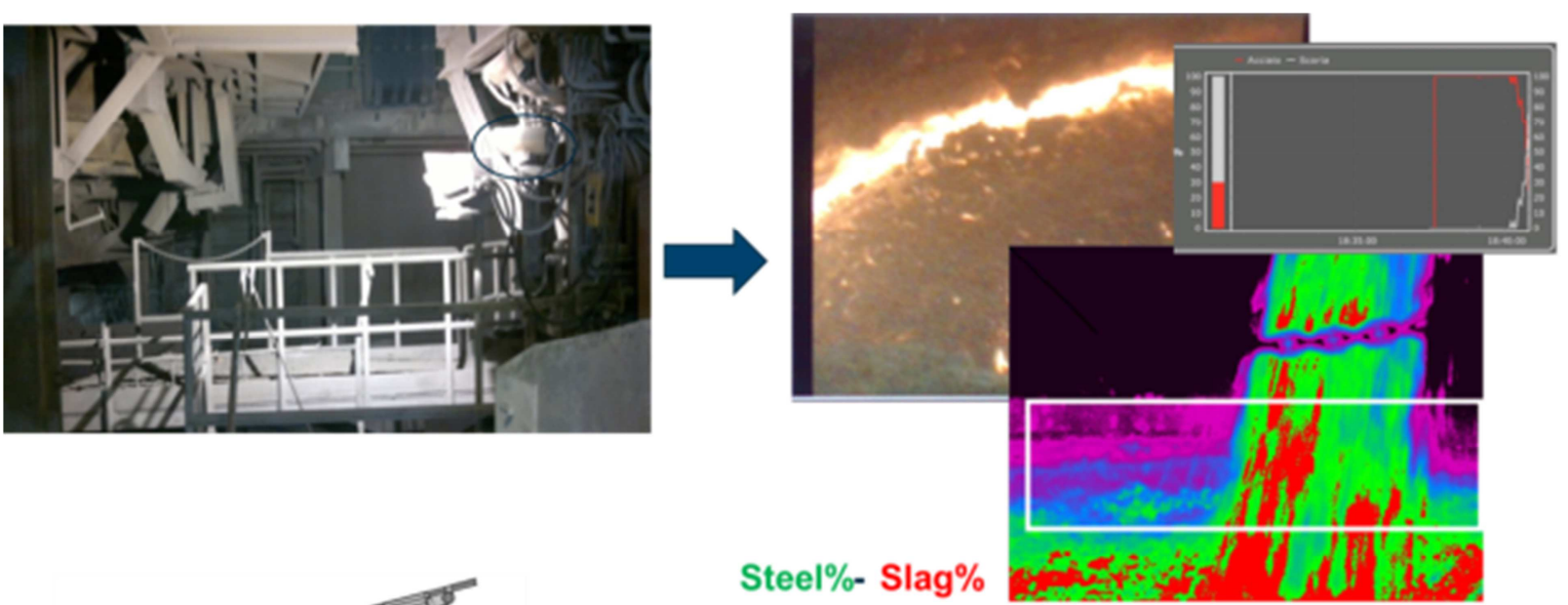

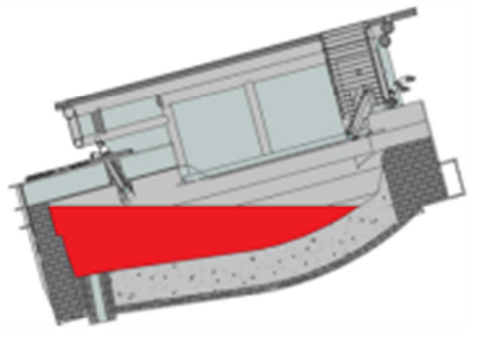

Automatic tilting angle control
Steel\%- Slag $\%$
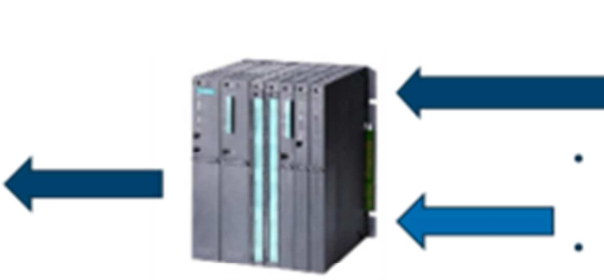

(from EAF weighing system) EBT life before repair (from Level 2)

Figure 5. Slag detection and control during tapping

The TAT system detects if slag is pouring out of the EBT during tapping and integrates the slag signal to calculate the amount of slag that is tapped in the ladle, allowing the operators to predict and improve the secondary metallurgy treatments.

\subsection{Automatic Cleaning and Sand Filling of the EBT}

After tapping is complete the operations of cleaning the EBT from obstruction and sanding are also automated. An extendable device with a camera is moved over the EBT area and inspection can be made remotely, to ensure there are no obstructions on the EBT, if all is clear, the EBT slide gate is closed and sand is poured over the EBT trough a conveying system, ensuring the proper amount is used on each filling.

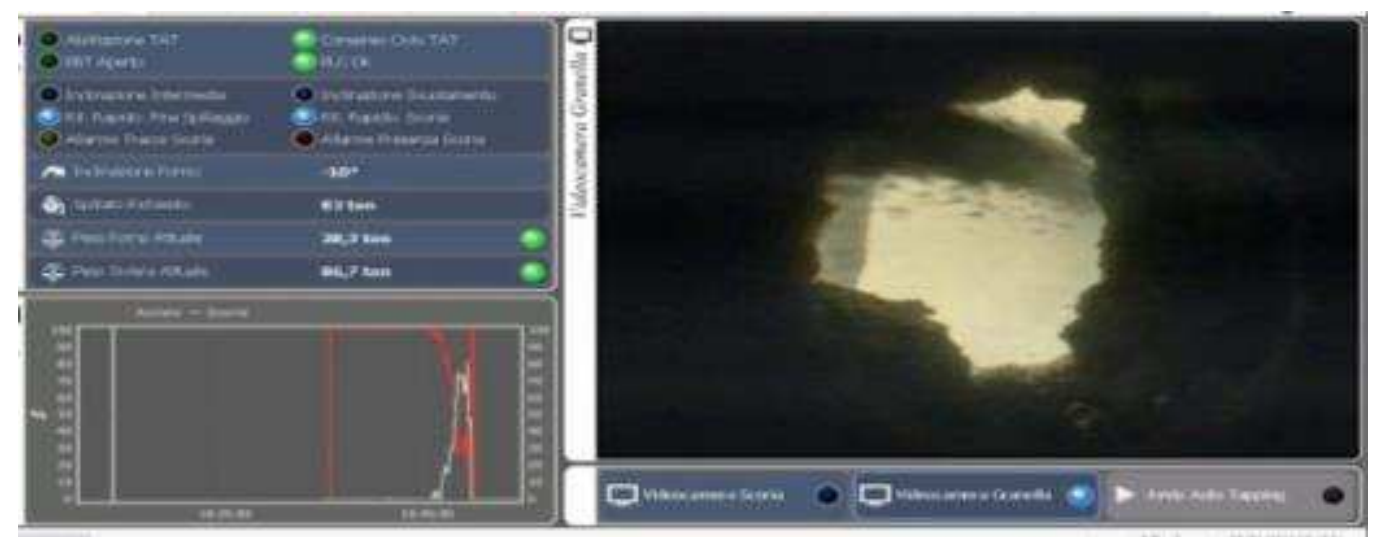

Figure 6. Video Camera monitoring the EBT sanding 


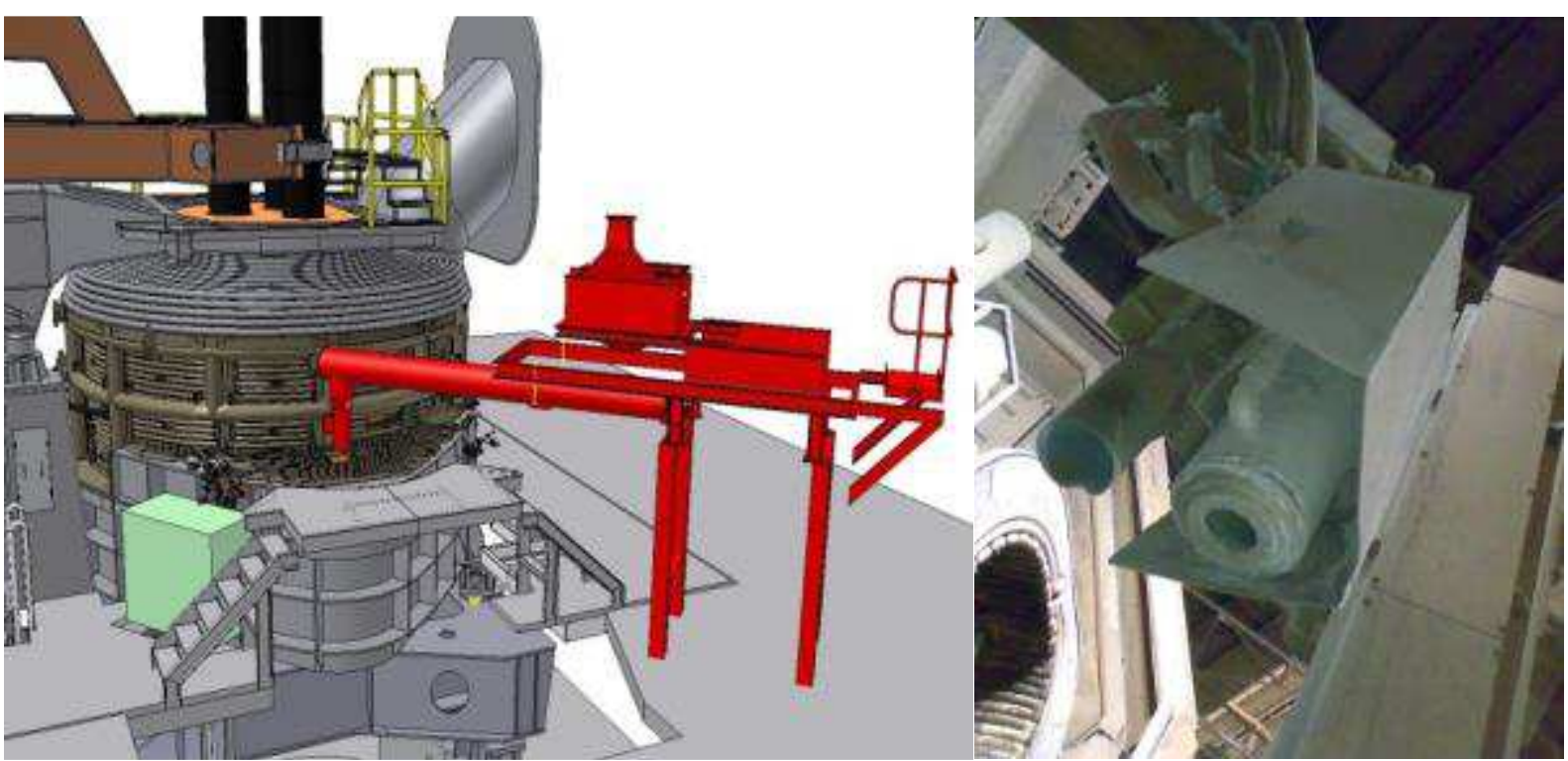

Figure 7. EBT automatic sanding equipment of the TAT

The clear benefit of these systems is the complete removal of the operator from these areas and minimizes delays and power off, by quickly clearing of obstructions in the EBT when they occur.

\subsection{Automatic Slag Door Cleaning (The Scraper)}

Conventional slag doors are typically water cooled panels that move up and down actuated by hydraulic cylinder, their function is to keep the tunnel area of the upper cage of the EAF closed, to retain foaming slag inside the EAF. This design however results in build ups of slag on the tunnel that eventually need to be cleared of the way to allow de-slaging of the furnace and also to allow for temperature and sample taking. This cleaning is typically done by mobile unit such as a bob cat or high lift, fitted with a pushing device and an operator driving it towards the furnace.

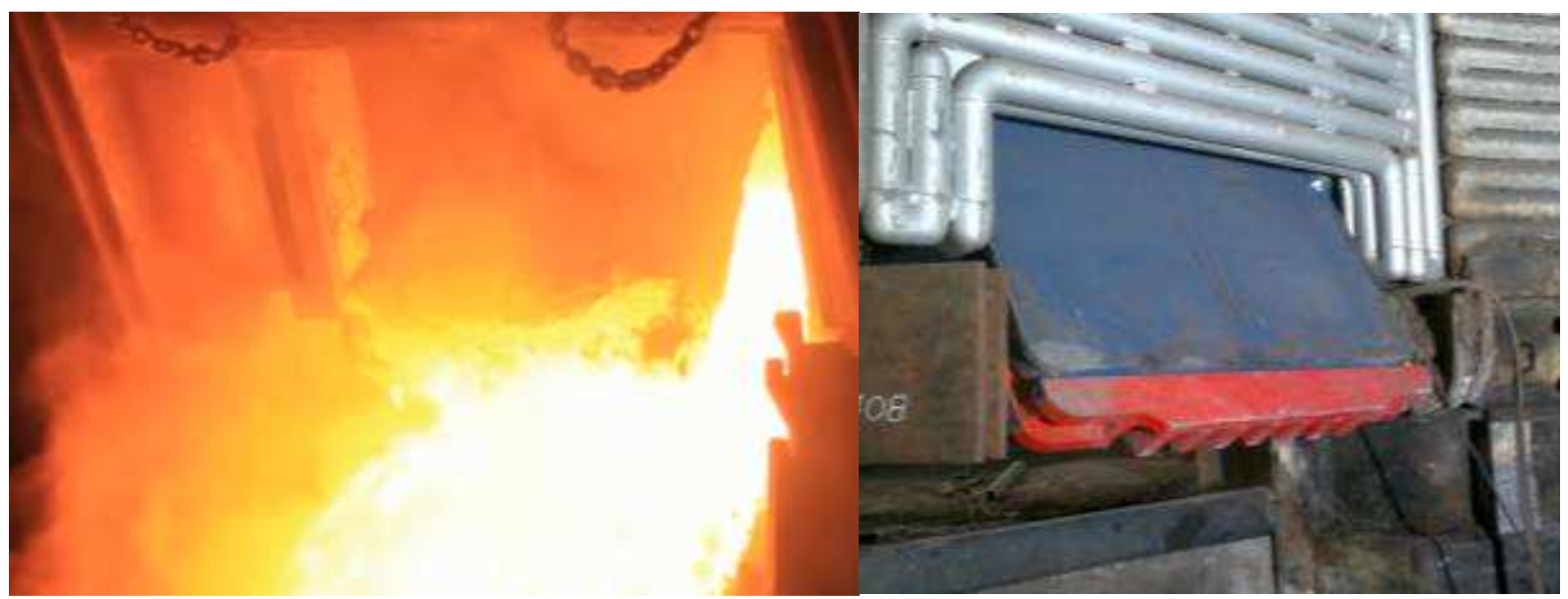

Figure 8. Door "scraper"

The automatic slag door is incorporated into the panel and tunnel design and its movement allows for tunnel and sill cleaning and levelling, scrap pushing, air tight door sealing without interferences to external door manipulators. 

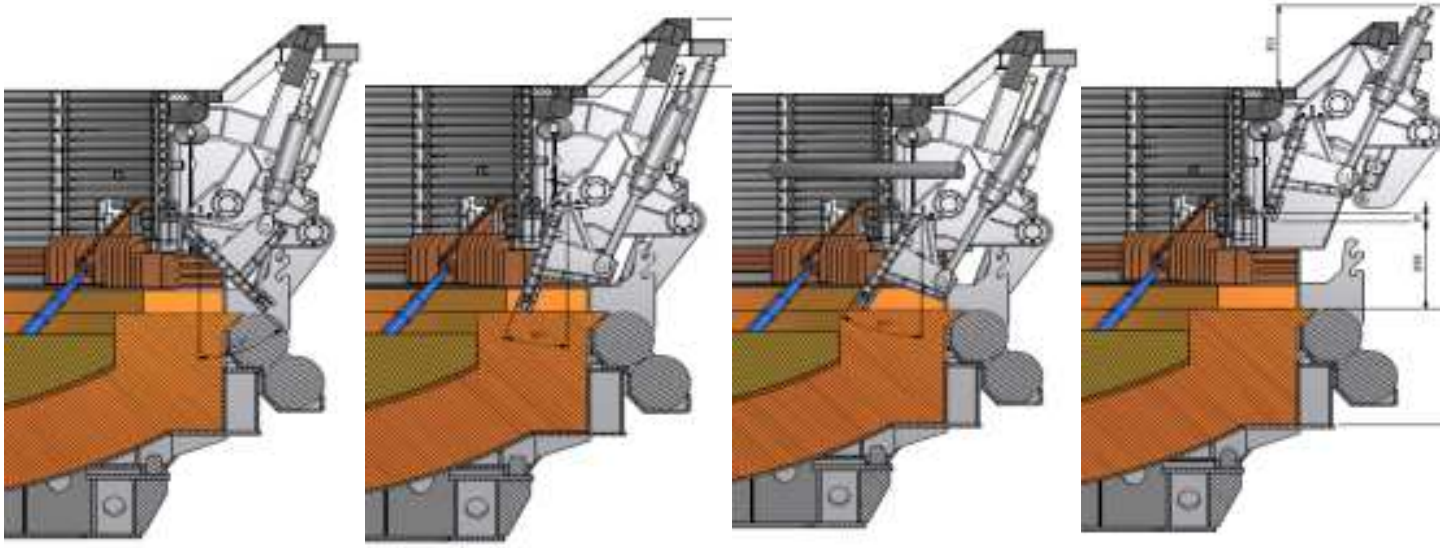

Figure 9. Scraper cleaning sequence

Keeping the slag door closed during operation, gives the following benefits:

- Improved productivity and energy saving

- Self-cleaning mechanism contributes to operator safety, as they are no longer exposed or operating a mobile equipment in front of the EAF

\subsection{Electrode Auto-slipping Device.}

On all EAF's, as the carbon electrodes wear out, electrode slipping operation is required. This is achieved by powering off the EAF, and through coordination of the EAF operator and crane operator, the electrode is slipped, by unclamping and clamping the electrode and moving the column, while being held by the crane, alternatively this can also be done by resting the electrode on the scrap, unclamping and raising the column, but this has a risk or electrode breakages.

Tenova's patented auto-slipping mechanism consist of a hydraulic clamp on top of the standard electro clamp of the arm that is able to clamp on to the electrode upper section and lower the electrode column trough the clamp to the appropriate length. This is achieved without the crane or resting the electrode on the scrap.

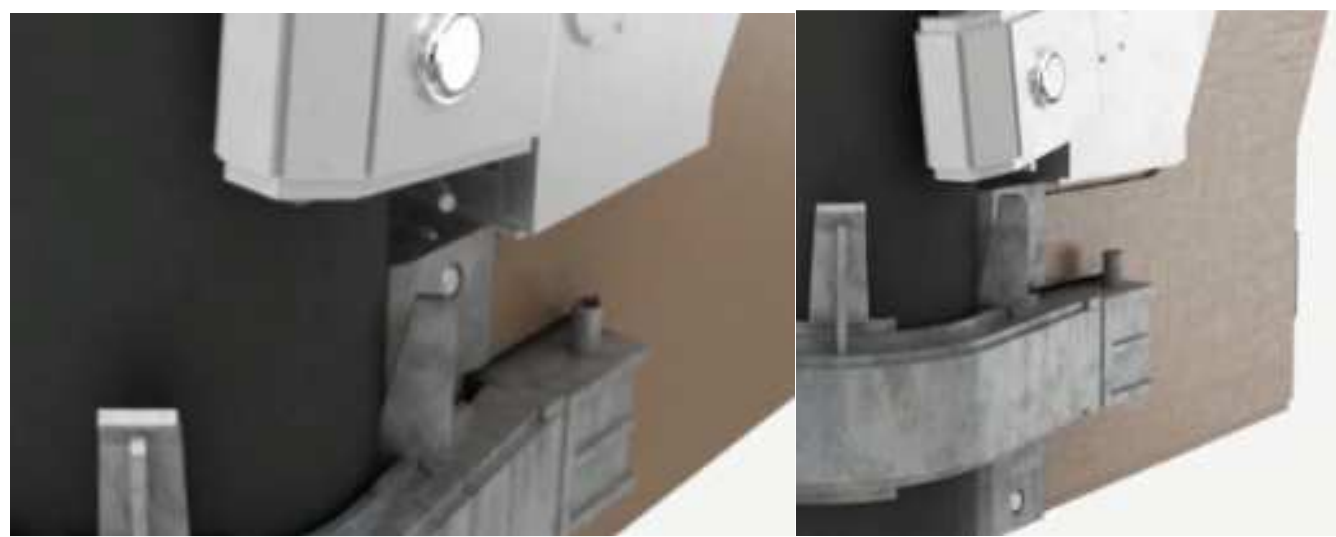

Figure 10. Elecrode auto-slipping device

The advantages of this system are:

- Maintain optimum triangulation of the electrode column below the clamp

- Reduce the Power off time for electrode slipping movements

- Increase safety of the electrode slipping operation

- Minimize electrode breakages 
- Saving up to 1 min per heat of power off

- Increasing average active power

- Reducing electrical power losses

\subsection{EAF Camera Monitoring System}

This system allows the panel inspection remotely; the operator can see the inside of the EAF from the control room. The camera is fixed outside the upper shell, on the top part of the panel, and is protected by a water cooled cover on the panel's window the cover opens by means of a small cylinder; allowing the camera to view inside the EAF during power off for refractory and panel inspection prior to the bucket charge. Typically three cameras allows to cover all interior of EAF panels
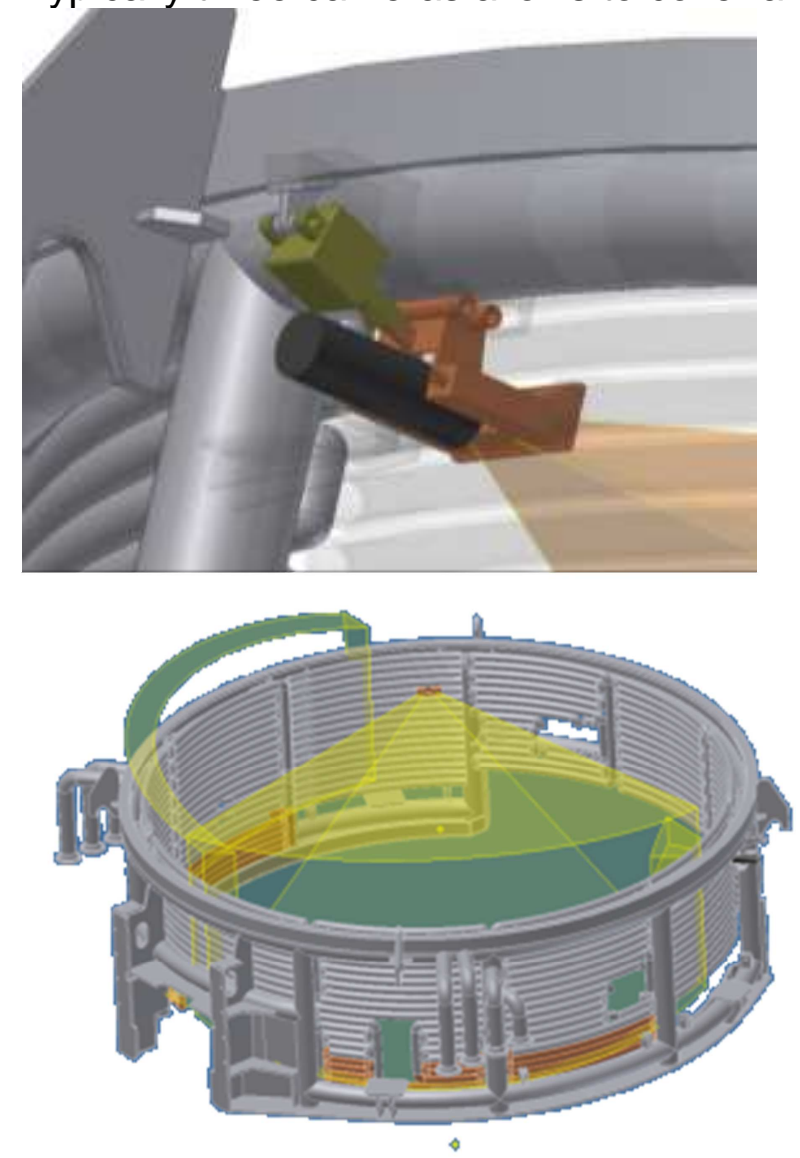

Figure 11. Location and area of influence of the monitoring cameras inside the EAF

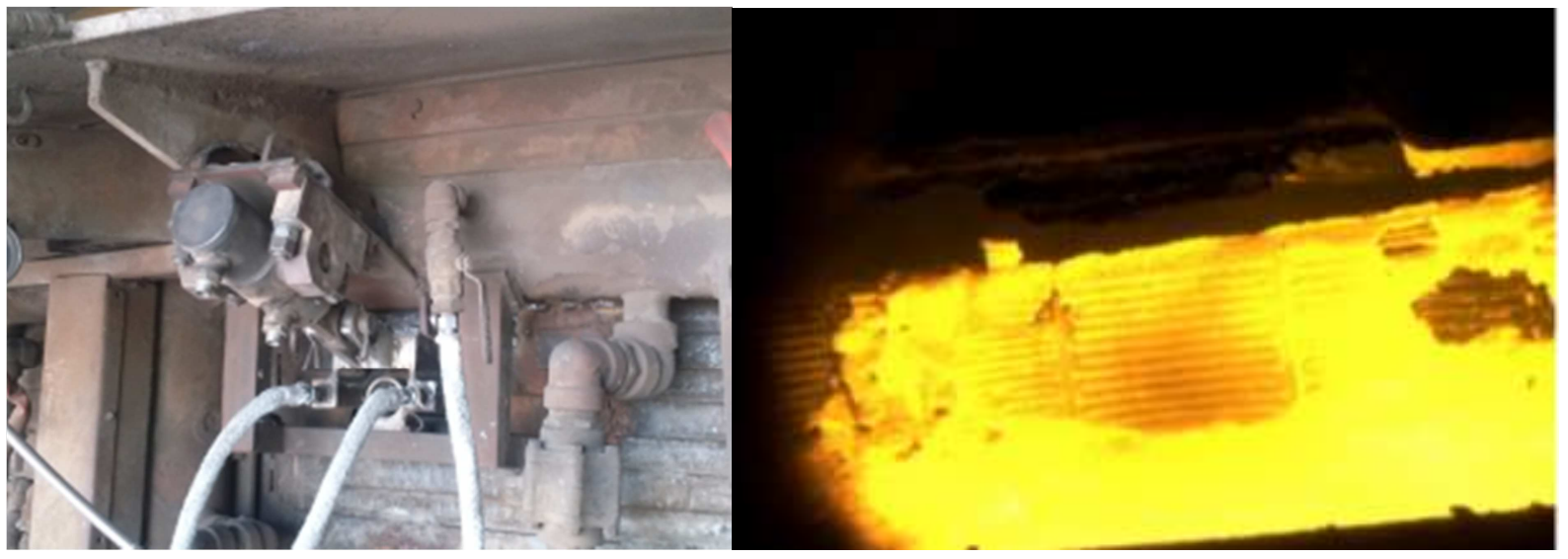


Figure 12. Actual location of the monitoring camera and image of inside the EAF before bucket charge

\subsection{PLTTM (Physical Ladle Tracking) System}

The physical ladle tracking system utilizes Radio Frequency Identification (RFID) technology to instantly detect and pinpoint the location of all the ladles in the melt shop in real time.

The RFID tag is attached to the ladle, and is protected by a special shield or armor. RFID readers and antennas are strategically located throughout the melt shop, and when the RFID tags are detected by the antennas, and a unique ID number associated to a particular ladle, is sent by the readers to a central server.

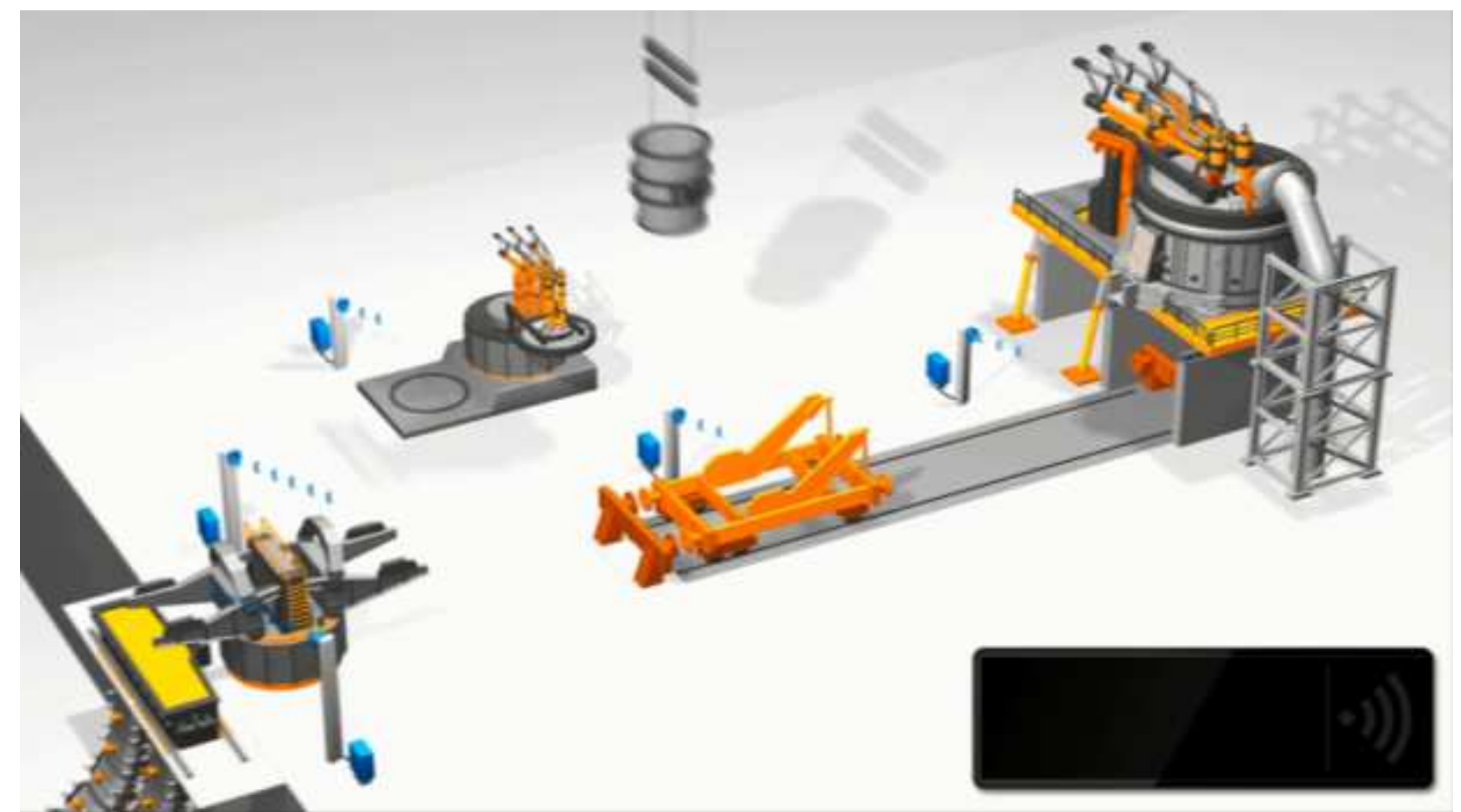

Figure 13. PLT system antennas located throughout the shop

Proprietary software then process the signals and displays current location as well as tracking residence time on each station, this allows for the creation of a database which can then be used on real time or as customized reports to:

- Detect the early deterioration of refractory in a ladle

- Maximize the life of the liquid steel ladles

- Reduce the heat losses and waiting time of a full ladle

- Determine the best refractory for a certain steel grade

- Determine the best practice

- Coordinate ladle tear out and repair times, ladle turnaround

- Define the best ladle to use in the cycle

- Compare and select different refractory supplier

\subsection{Automatic Scrap Charge}

The Automatic Scrap Charge system starts from the formation of the bucket itself. The System foresees an HMl where the Furnace Chief organizes the Heat sequence on a production Plan page. Based on that, the crane operator in the scrap yard automatically receive Recipe for the bucket preparation on the crane panel PC. 

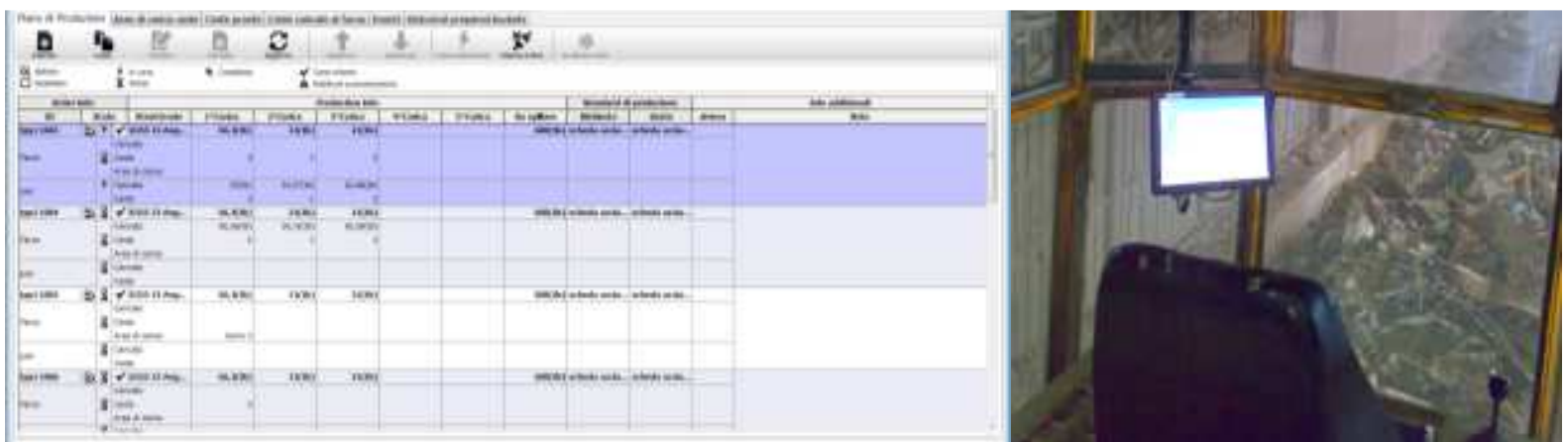

Figure 14. Scrap yard production page and panel PC in the crane cabin

Scrap Yard Operators are constantly up to date with the actual furnace situation. By means of the panel PC in the crane, the crane operator can add notes and confirm that the bucket has been prepared according to the recipe or can modify the scrap mix according to the actual scrap grades charged for that specific bucket. Once the bucket is prepared, it is made available to the EAF charge crane. By means of RFID tags, the Bucket is identified by the EAF automation and the actual bucket recipe is incorporated in the EAF mass and energy balance model that is controlling the EAF operation. The system also records the sequence of crane material discharged. This feature helps the process manager to control if the crane operator follows the correct material layering.

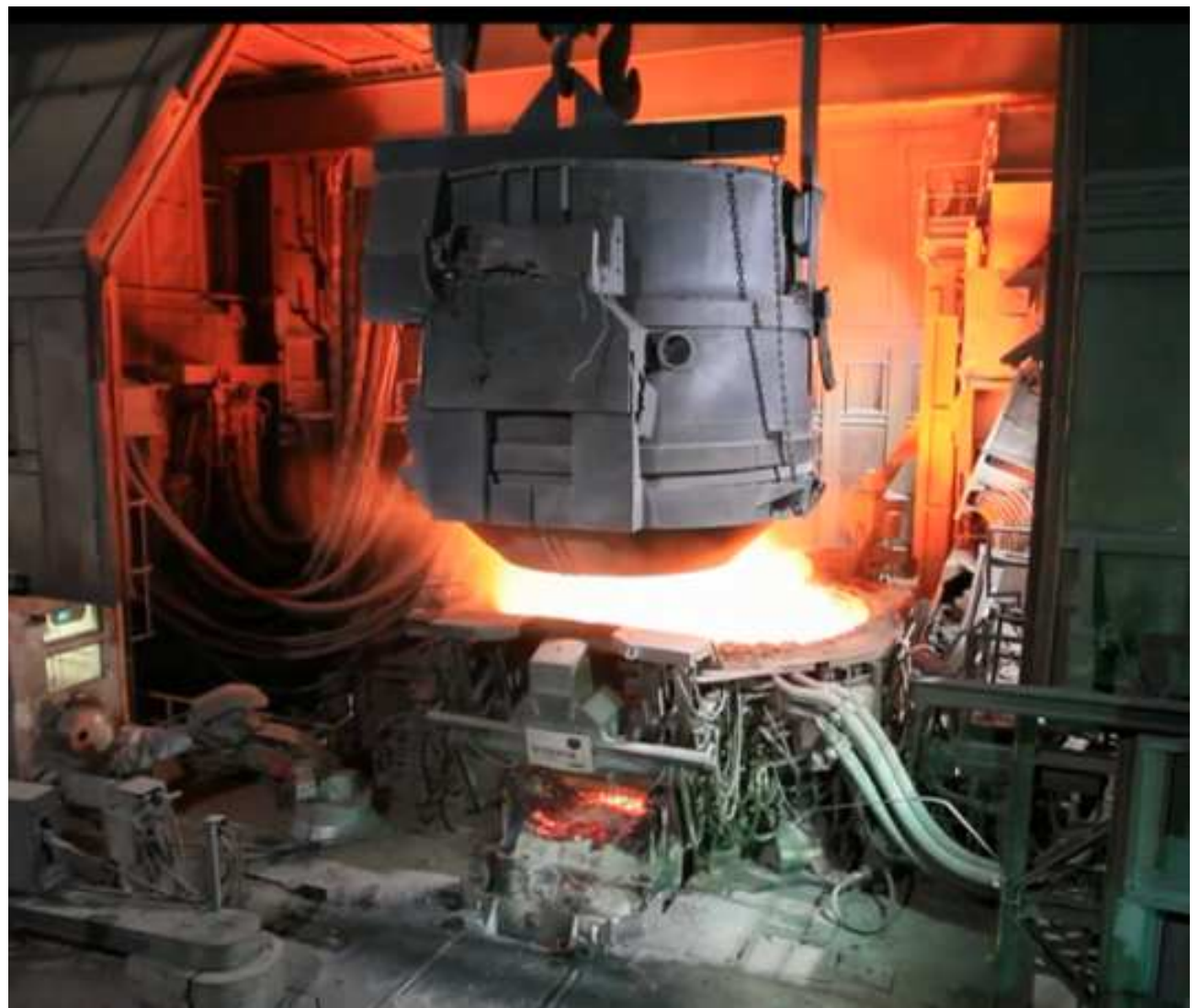

Figure 15. Scrap bucket automatically charged to the EAF without the need of a crane operator

By means of limit switches, once the EAF is ready to receive the scrap bucket, the EAF charge crane moves to the designated positions in order to minimize the roofopen time and charge as quick as possible the scrap bucket into the EAF. The automatic position, the fact that the bucket is moving in accordance to the movement 
of the EAF roof in a controlled and tested way, so to avoid interferences and the fact that the positions are always kept the same by limit switches, makes the scrap charge operation consistent and controlled, minimizing power off time and energy losses.

\section{CONCLUSION}

The integration of these technologies - all tested, implemented and operating in various steel plants in Europe and United States - contribute to the optimization and safety of EAF melting process, by ensuring repetitiveness of the operation, providing additional control and monitoring tools and in some cases, eliminating human error altogether, while at the same time, significantly improving the safety of the operators by reducing their exposure to high risk activities on to the shop floor.

The role of the operator becomes more involved with technical tracking of variables, process control and coordination with the rest of the melt shop, than physical labor. 